\title{
Validación de la escala de evaluación del significado del cuidado
}

\section{Validation of scale of assessment of significance of caring}

\author{
Validação da escala de avaliação do significado do cuidar
}

Rosa Aparecida Pavan Bison ${ }^{1}$; Debora Vieira Almeida²; Jair Licio Ferreira Santos³; Antonia Regina Ferreira Furegato ${ }^{4}$

${ }^{1}$ Escola de Enfermagem da Universidade de São Paulo de Ribeirão Preto Brasil. Enfermeira, doutora em enfermagem psiquiátrica pela EERP/USP. rosa.bison@unimedararas.com.br

${ }^{2}$ Centro Universitário São Camilo, São Paulo - SP.Brasil. Enfermeira, mestre e doutora pela Escola de Enfermagem da Universi-

dade de São Paulo, pós-doutorando em Bioética pelo Centro Universitário São Camilo. deboravalmeida@gmail.com

${ }^{3}$ Faculdade de Medicina de Ribeirão Preto/ USP. Brasil. Professor Titular Sênior do Departamento de Medicina Social da Faculdade de Medicina de Ribeirão Preto/ USP. jairlfs@fmrp.usp.br

${ }^{4}$ Escola de Enfermagem de Ribeirão Preto/ USP. Brasil. Enfermeira, Professora Titular Sênior do Departamento de Enfermagem Psiquiátrica e Ciências Humanas da Escola de Enfermagem de Ribeirão Preto/ USP. furegato@eerp.usp.br

Cómo citar este artículo en edición digital: Pavan Bison, R.A; Almeida, D.V; Ferreira Santos, J.L; Ferreira Furegato, A.R. (2013)

Validación de la escala de evaluación del significado del cuidado Cultura de los Cuidados.17, 37. Disponible en: http://dx.doi. org/10.7184/cuid.2013.37.09

Correspondencia: Rosa Aparecida Pavan Bison. Escola de Enfermagem da Universidade de São Paulo de Ribeirão Preto Brasil. Correo electrónico: rosa.bison@unimedararas.com.br

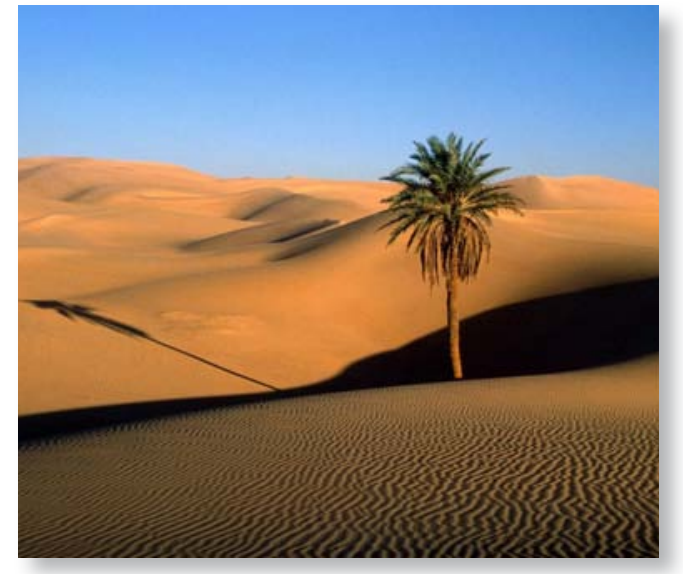

\section{ABSTRACT}

Following Morse benchmark, the Scale for the Assessment of Significance of Care (SASC) received a construct validation in 2003. This present paper aimed to continue the process of validation of the instrument by assessing its internal consistency and reproducibility. To estimate internal consistency it was used the original 2003 information to calculate the values of Cronbach's Alpha in each of the five areas and for each question. Appraisal of Reproduci- bility was done through test-retest technique: the scale was administered to 98 subjects, all student nurses. The reproducibility was measured by the degree of agreement between the two measurements using the percentage of observed agreement, the intraclass correlation coefficient and the coefficient of reliability of Spearman-Brown. It was concluded that it is a consistent instrument with good reproducibility that can contribute to the knowledge of the attitudes of nurses in relation to care.

Key Words: nursing care; validation studies; attitude; education, nursing; concept formation.

\section{RESUMEN}

De acuerdo a la referencia de Morse, la Escala de Evaluación del Significado del Cuidado (EESC) recibió validación de constructo en 2003. El presente trabajo tiene el objetivo de continuar el proceso de validación del instrumento: evaluación de consistencia interna y reproducibilidad. Consistencia interna: se 
utilizó la información original de 2003 para el cálculo de los valores de Alfa de Cronbach en cada una de las cinco áreas y para cada pregunta. Reproducibilidad: se realizó mediante la técnica de test-retest. La escala se administró a 98 sujetos, estudiantes de enfermería. La reproducibilidad se midió por el grado de concordancia entre las dos mediciones, a través de medidas que compiten: el porcentaje de acuerdo observado, el coeficiente de correlación intraclase y el coeficiente de fiabilidad de Spearman-Brown. Se concluyó que es un instrumento consistente, con buena reproducibilidad y que puede contribuir al conocimiento de las actitudes de las enfermeras en relación al cuidado.

Descriptores: Atención de Enfermería; Estudios de Validación; Actitud; Educación en Enfermería; Formación de Concepto.

\section{RESUMO}

Seguindo o referencial de Morse, a Escala para Avaliação do Significado do Cuidar (EASC) recebeu validação de constructo, em 2003. No presente trabalho, objetivou-se completar o processo de validação do instrumento: avaliação da consistência interna e da reprodutividade da EASC. Para estimar a consistência interna foram usadas as informações originais do estudo sendo calculados os valores de Alfa de Cronbach para cada um dos 5 domínios e de cada questão. Para a reprodutibilidade, através da técnica de teste e reteste, a escala foi aplicada a 98 sujeitos, estudantes de enfermagem. A reprodutividade foi medida pelo grau de concordância entre as duas aferições, através de medidas concorrentes: as porcentagens de concordância observadas, o coeficiente de correlação intraclasse e o coeficiente de confiabilidade de Spearman-Brown. Concluiu-se que se trata de um instrumento consistente e de boa reprodutividade que poderá contribuir para o conhecimento das atitudes dos enfermeiros em relação ao cuidar.

Descritores: cuidados de enfermagem; estudos de validação; atitude; educação em enfermagem; formação de conceito.

\section{INTRODUÇÃO}

O conceito de cuidado profissional tem sido estudado sob as perspectivas técnicas, científicas, éticas e filosóficas. O cuidado ao ser humano é reconhecido pela enfermagem não apenas como uma meta, mas como a essência da prática e do ensino da profissão, tanto no aspecto gerencial como do cuidado direto à saúde das populações. $\mathrm{O}$ cuidado deve ser entendido como um processo de facilitação através do qual o enfermeiro cria as condições favoráveis para que a pessoa faça suas escolhas, visando a resolução dos seus problemas de saúde. Na visão humanista, o cuidado do outro requer uma atitude ética fundamental, um modo de agir onde a pessoa desvincula-se de si própria e centra-se no outro, com desvelo e solicitude, visando o bem e a dignidade de cada um. A enfermagem, nas mais diferentes especialidades, tem dedicado seu trabalho nesta direção (Bison, Furegato e Santos, 2007; Peplau, 1993; Shirataki et al, 2003; Freeth, 2007; Almeida, 2012; Furegato e Morais, 2009; Bradley e Falk-Rafael, 2011; Waldow, 1999; Vielva, 2002; Christovam, Porto e Oliveira, 2012).

Partindo-se do princípio de que as ações da enfermagem são terapêuticas quando auxiliam o indivíduo doente e/ou necessitado de cuidados de saúde, objetiva e subjetivamente, nos mais diferentes procedimentos técnicos e científicos, o enfermeiro apresenta-se como um agente preparado para reconhecer a necessidade de ajuda e a ela responder adequadamente. Portanto,pressupõe-se que o indivíduo seja o 
foco do cuidado e a enfermagem seja o instrumento para auxiliá-lo nas suas necessidades.

\section{REFERENCIAL TEÓRICO}

Como base teórica adotou-se conceitualmente as propostas da enfermeira e teorista Morse que explica o cuidado através de cinco classificações ontológicas: a) Cuidar como característica humana; b) Cuidar como imperativo moral ou ideal; c) Cuidar como afeto; d) Cuidar como relação interpessoal; e) Cuidar como ação terapêutica (Morse, 1991; Morse, 1995).

Nessa perspectiva, realizou-se, em 2003, um estudo focalizando os significados e significantes do cuidado e suas correlações com o processo do cuidar, visando a construção de um instrumento para se conhecer a atitude do enfermeiro em relação ao cuidado, compreendendo-se atitude como uma propriedade psicológica que reflete uma disposição para a ação (Pasquali, 2010).

A escala sobre o significado do cuidado de enfermagem foi construída após densas análises, revisões bibliográficas e reflexões sobre a realidade da profissão, tendo por base a experiência dos autores com relação aos construtos do instrumento de medida. Após uma série de procedimentos metodológicos, o instrumento EASC com cinco domínios (acompanhando as cinco classificações ontológicas de Morse) obteve validação de seu construto, totalizando, até esta etapa de validação, 45 itens. Este instrumento foi aplicado em uma pesquisa da qual participaram 171 sujeitos (alunos, enfermeiros da prática e docentes), verificando-se que embora os escores, no seu conjunto, fossem muito próximos, havia diferenças de concepções entre alunos e professores, especialmente nas categorias "característica pessoal" e "imperativo moral" (Bison, Furegato e Santos, 2007).
Essas diferenças também foram evidenciadas em pesquisa publicada em 2010, que teve como sujeitos de pesquisa apenas enfermeiros (assistenciais e mestres) de uma clínica de Cuba utilizando a EASC (Vergel, Betancourt e Guerra, 2010).

$\mathrm{Na}$ investigação atual, nos propusemos a concluir duas importantes etapas para finalizar a validação deste instrumento: avaliar sua confiabilidade interna e seu potencial de reprodutibilidade.

Portanto, a pesquisa atual teve por objetivo estimar a confiabilidade da Escala de Avaliação do Significado do Cuidar (EASC) por meio da validação da sua consistência interna e da sua reprodutibilidade.

\section{MÉTODO}

\section{Consistência interna}

Para estimar a consistência interna do instrumento foram usados os dados originais do estudo com os 171 sujeitos da pesquisa (Bison, Furegato e Santos, 2007). Foram calculados os valores do coeficiente Alpha de Cronbach para cada um dos cinco domínios e para a escala como um todo. Como tem sido a prática nestes casos, foram considerados aceitáveis os valores do coeficiente Alpha superiores a 0,60 . Foram igualmente calculados os valores de Alpha referentes à eventual eliminação de cada questão. Um aumento superior a $5 \%$ no valor de Alpha, obtido após a eliminação foi tomado como indicativo para a eventual exclusão da questão, desde que sua ausência não prejudicasse de forma veemente o arcabouço teórico de Morse utilizado na construção do instrumento.

\section{Reprodutibilidade}

A reprodutibilidade foi medida pelo grau de concordância entre as duas aferições, atra- 
vés de várias medidas concorrentes: as porcentagens de concordância observada; o coeficiente de correlação intraclasse; o coeficiente de confiabilidade de Spearman - Brown (Winer, Brown e Michels, 1991).

A coleta de dados desta etapa da pesquisa ocorreu de 26 de março a 07 de maio de 2012. Participaram 98 estudantes do curso de enfermagem da Universidade Federal do Triângulo Mineiro (UFTM), maiores de 18 anos e que cursavam o primeiro, terceiro, quinto e sexto períodos do curso. Trata-se de uma amostra por conveniência devido a facilidade de reunir grande número de pessoas para teste e reteste do material. Foram eliminados 12 sujeitos porque não estavam presentes no dia da realização do reteste, permanecendo 86 indivíduos nas duas fases.

Uma das pesquisadoras foi até as salas de aula, explicou os objetivos da pesquisa, esclareceu as dúvidas e informou que haveria uma segunda aplicação do instrumento de 15 a 30 dias da primeira coleta e que não se tratava de aferir a capacidade das pessoas entrevistadas em recordar as respostas da primeira aplicação, mas sim que as técnicas de análise permitiriam avaliar a adequação do instrumento para obter respostas semelhantes em ocasiões distintas. Foi ainda informado que a identificação dos sujeitos na primeira aplicação era realizada apenas com o objetivo de garantir o pareamento para a segunda aplicação. O anonimato foi assegurado a partir da segunda aplicação, quando apenas o número do questionário permaneceu como informação. Os alunos que aceitaram participar receberam o Termo de Consentimento Livre e Esclarecido (TCLE), assinaram e depois responderam a EASC.

Projeto aprovado pelo Comitê de Ética em Pesquisa da UFTM (Parecer n²169).

\section{RESULTADOS}

\section{a) Análise da consistência interna}

Todos os valores do coeficiente Alpha foram superiores a 0,60. Em particular, o coeficiente para todas as questões consideradas em conjunto foi superior a 0,70. Estes resultados constam da tabela 1 , na qual também constam os valores que seriam obtidos para Alpha, caso algumas das questões fossem eliminadas. As questões escolhidas são aquelas que, em cada domínio, provocariam o maior acréscimo relativo ao Alpha original. (TABELA 1)

Após as análises, as questões passíveis de remoção da Escala foram: 03-Exercer a enfermagem é uma atividade difícil; 08- Ao cuidar, o enfermeiro deve ser imparcial; 28-Educação deve fazer parte do cuidado de enfermagem; 34-Habitualmente, utilizo o recurso da auto-medicação; 35-Quem cuida precisa ter virtudes especiais tais como responsabilidade, respeito e honestidade.

Verificou-se que as afirmativas 3 e 34, quando excluídas do instrumento, levariam a aumentos na consistência dos respectivos domínios, superiores a 5\%. Optou-se por eliminar apenas a questão 3, pela sua menor importância no arcabouço teórico do instrumento.

Os resultados finais da análise de consistência interna constam na tabela 2 , com a exclusão da questão 3 do domínio 2. (TABELA 2)

Observa-se que todos os valores de alpha, em cada domínio, são superiores a 0,60, denotando boa consistência interna. O resultado na análise entre os cinco domínios apresenta um valor pobre para a consistência $(0,45)$, o que é coerente e indica, como deveria se esperar, a representação de dimensões diferentes por domínio. Ao contrário, se existisse grande consistência entre os 5 domínios estaríamos frente a uma indicação de unidimensionalidade, isto é, os domínios não estariam abrigando 


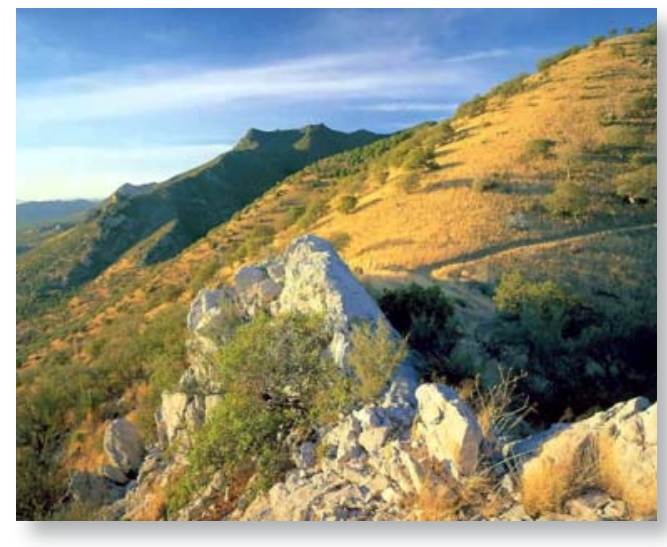

dimensões distintas entre si.

A EASC final contém 44 questões assim distribuídas, em cada domínio:

1-O cuidado como característica pessoal humana: 1, 3, 5, 6, 10, 13, 29, 34, 40;

2-O cuidado como imperativo moral: 9, $14,16,21,32,35,39,44$;

3 -O cuidado como afeto: $2,7,8,15,20,22$, $28,38,42$;

4-O cuidado como relação interpessoal: 4 , $17,18,23,24,26,33,36,43$;

5-O cuidado como intervenção terapêutica: 11, 12, 19, 25, 27, 30, 31, 37, 41.

\section{b) Análise de reprodutibilidade}

Perdas de informações: na aplicação do instrumento no reteste, 12 indivíduos não responderam às questões na segunda coleta de dados, o que ocasionou a eliminação dos mesmos também na fase teste. Permaneceram, portanto, 86 indivíduos com respostas nas duas fases, com uma perda de $12,2 \%$.

Respostas faltantes: na primeira fase do teste-reteste, 8 indivíduos deixaram de responder a uma das questões; na segunda fase, 4 sujeitos omitiram uma das respostas. Em volume de informações estas omissões representam muito pouco: $0,2 \%$ no primeiro caso e $0,1 \%$ no segundo. No entanto, como as técnicas de avaliação não admitem respostas em branco, uma possibilidade seria a eliminação desses respondentes, o que representaria uma perda adicional de 14\%. Diante desse dado, resolveu-se proceder à imputação das respostas faltantes por meio da média dos valores respondidos pelo indivíduo no domínio no qual se situava a resposta omitida.

Os resultados podem ser observados nas primeiras colunas da tabela 3 , onde encontram-se os domínios e as porcentagens de concordância, observadas entre os valores do teste e do reteste. Nota-se que é alto o nível de concordância, com um mínimo de $94,3 \%$. Os coeficientes de correlação intraclasses foram calculados através de análise de variância e estimam o limite superior da proporção da variabilidade total do escore que é explicada pela variação dentro dos testes e não entre indivíduos.

Além disso, o coeficiente de Spearman Brown mede a confiabilidade depositada na média das medidas. Em todos os domínios, observa-se que houve bons níveis de correlação e altos valores de confiabilidade nos escores. (TABELA 3)

\section{DISCUSSÃO}

Um instrumento tal como a EASC - Escala de Avaliação do Significado do Cuidar procura verificar o grau de adesão do indivíduo a uma série de afirmações que sejam expressões de algo favorável ou desfavorável em relação a um objeto psicológico. O conjunto de afirmações focaliza uma atitude tendo por base três grandes vertentes: teórica, empírica e analítica (Pasquali, 2010).

Os resultados desta pesquisa mostraram que esta escala apresenta bons indicadores de validade de construto, e, devido às suas propriedades psicométricas, apresenta correlações significativas entre as variáveis. Sendo 
multifatorial, a Escala de Avaliação do Significado do Cuidar (EASC) permite avaliar, com escores de medida independentes, diferentes dimensões do cuidar: característica humana, imperativo moral ou ideal, afeto, relação interpessoal e ação terapêutica. Com estas cinco dimensões observa-se que existe estreita relação entre a enfermagem e a humanização da assistência preconizada como direito de todos.

Cuidar do outro é uma das razões pelas quais as pessoas escolhem uma profissão da área da saúde. Ao exercer a sua profissão os conflitos de interesses podem dificultar o pleno exercício dos direitos de quem cuida e de quem é cuidado o que vem reforçar os conceitos éticos e de cuidados como atividade terapêutica (Bison, Furegato e Santos, 2007; Peplau, 1993; Shiratori et al, 2003; Waldow, 1999; Gonzalez e Ruiz, 2011; Shoeller, Leopardi e Ramos, 2011).

As políticas públicas definem leis e normatizações para atender as necessidades das pessoas e as demandas da coletividade. Felizmente, as propostas de humanização da assistência, tanto em nível nacional como internacional, reforçam as dimensões do cuidar propostas por Morse, sustentadas por valores de respeito aos direitos e à autonomia com a participação ativa dos sujeitos em todas as instâncias do processo saúde/doença. Em conformidade com as políticas internacionais de defesa dos direitos do cidadão, o Ministério da Saúde brasileiro preconiza que se incluam nas ações em saúde os diferentes sujeitos que participam desde suas singularidades, no planejamento, na implementação e na avaliação dos processos de produção de saúde e de formação do trabalhador de saúde, com vistas a humanizar as práticas de gestão e de cuidado (Brasil, 2010).
A experiência dos autores na construção e validação deste instrumento, assim como a participação direta de alunos e enfermeiros na amostragem (tanto na primeira fase de validação do construto como na segunda fase de validações da sua consistência e reprodutividade) permitem afirmar que a EASC configura-se como um instrumento de medida adequado para se avaliar o significado atribuído pelos enfermeiros ao ato de cuidar.

As informações obtidas com este instrumento poderão contribuir para a qualidade do atendimento prestado aos pacientes, pois oferece indicadores das várias dimensões do cuidado de enfermagem, no desempenho de suas atribuições junto às equipes de atenção à saúde das populações.

Os resultados desta pesquisa contribuem para reforçar o poder de avaliação da atitude do enfermeiro em relação à assistência de enfermagem no cuidado da pessoa que precisa de cuidados preventivos, curativos ou de reabilitação em saúde.

Ressalta-se que a eliminação da questão de número 3 do instrumento original não afeta o desenho teórico sob o qual foi construído e traz melhorias importantes para sua consistência interna.

\section{CONCLUSÕES}

Os resultados das aplicações dos testes para validação do instrumento Escala de Avaliação do Significado do Cuidar (EASC) demonstraram que trata-se de um instrumento consistente e de boa reprodutibilidade.

A escala original já foi utilizada, antes dos atuais procedimentos de validação, com resultados expressivos. Futuras investigações poderão confirmar sua eficácia e trazer novos resultados que contribuirão para melhorar o cuidado de enfermagem. 


\section{REFERÊNCIAS}

- Almeida, D.V. (2012) A humanização dos cuidados em saúde: uma proposta conceitual. AB Editora, Goiânia.

- Bison, R. A. P; Furegato, A. R. .; Santos, J. L. F. (2007) La percepción del cuidar entre estudiantes y profesionales de enfermeria. Index Enferm. 16(56):30-4. Disponível http://scielo.isciii.es/scielo.php?script=sci_arttext\&pid $=$ S1132-12962007000100007. baixado 20 de junho de 2013.

- Bradley, P; Falk-Rafael, A. (2011) Instrumental care and human-centered caring: rhetoric and lived reality. Adv. Nurs. Sc. 34(4):297-314.

- Brasil. Ministério da Saúde. Secretaria de Atenção à Saúde. Política Nacional de Humanização. (2010) Série B. Textos Básicos de Saúde - Cadernos HumanizaSUS, Brasília: Ministério da Saúde.

- Christovam, B. P; Porto, I. S; Oliveira, D. C. (2012) Gerência do cuidado de enfermagem em cenários hospitalares: a construção de um conceito. Rev. Esc. Enferm. USP. 26(3):734-41.

- Freeth, R.. (2007) Humanising psychiatry and mental health care: the challenge of the person-centred approach. Radcliffe Publishing, Oxford.

- Furegato, A. R. F; Morais, M. C. (2009) Bases do relacionamento interpessoal em enfermagem. In: Programas de Atualização em Enfermagem - PROENF: Saúde do adulto, Ciclo 4, Modulo 1. Artmed. Porto Alegre: 45-73.

- Siles, J; Ruiz, M. C. S. (2011) A história cultural e a estética dos cuidados de enfermagem. Rev. Latino-Am. Enferm. 19(5):1096-105. Disponível http://www. scielo.br/scielo.php?script $=$ sci_arttext $\&$ pid $=$ S0104$-11692011000500006 \& \operatorname{lng}=$ pt\&nrm $=$ iso\&tlng=pt. baixado 20 de junho de 2013.

- Morse, J. M. (1991) Comparative analysis of conceptualizations and theories of caring. J. Nurs. Schol. 23(2):11926.

- Morse, J. M. (1995) Exploring the theoretical basis of nursing using advanced techniques of concept analysis. Adv. Nurs. Sc. 17(3):31-46.

- Pasquali, L. (2010) Instrumentação psicológica: fundamentos e práticas. Artmed, Porto Alegre.
- Peplau, H. E. (1993) Relaciones interpersonales en enfermería. Ed. Científicas y Técnicas, Barcelona.

- Schoeller, S. D.; Leopardi, M. T.; Ramos, F. S. (2011) Cuidado: eixo da vida, desafio da enfermagem. Rev. Enferm. UFSM. 1(1):88-96.

- Shiratori, K.; Figueiredo, N. M. A.; Porto, F.; Silva, C. S. I.; Teixeira, M. S. (2003) O sentido de ser humano: uma base reflexiva para o cuidado de enfermagem. Rev. Enferm. UERJ. 11(2):212-16.

- Vergel, L. L. H.; Betancourt, D. M. Z.; Guerra, A. J. M. (2010) La percepción del cuidado en profesionales de enfermería. Rev. Cubana Enferm. 26(1):30-41. Disponível http://scielo.sld.cu/scielo.php?pid=S0864-03192010000100007\&script=sci_arttext. baixado $20 \mathrm{de}$ junho de 2013.

- Vielva, J. (2002) Ética profesional de la enfermería. Editorial Descleé De Brouwer, Bilbao.

- Waldow, V. R. (1999) Cuidado Humano: o resgate necessário. Sagra Luzzatto, Porto Alegre.

- Winer, B. J.; Brown, D. R.; Michels, K. M.(1991) Statistical principles in experimental design. 3. McGraw-Hill, New York: 
Tabela 1 - Valores alpha de Cronbach obtidos na análise do instrumento que avalia o significado do cuidar Uberaba, MG, 2012

\begin{tabular}{|l|ccll|}
\hline DOMíNIO & $\begin{array}{l}\text { Sem } \\
\text { eliminação }\end{array}$ & $\begin{array}{l}\text { Com } \\
\text { eliminação }\end{array}$ & $\begin{array}{l}\text { Item } \\
\text { eliminado }\end{array}$ & $\begin{array}{l}\text { Aumento } \\
\text { percentual } \\
\text { obtido pela } \\
\text { eliminação }\end{array}$ \\
\hline $\begin{array}{l}\text { 1- O cuidado como } \\
\text { característica pessoal } \\
\text { humana }\end{array}$ & 0,62 & 0,63 & Q35 & 1,6 \\
$\begin{array}{l}\text { 2- O cuidado como } \\
\text { imperativo moral }\end{array}$ & 0,66 & 0,70 & Q3 & 6,1 \\
$\begin{array}{l}\text { 3- O cuidado como afeto } \\
\begin{array}{l}\text { 4- O cuidado como } \\
\text { relação interpessoal }\end{array}\end{array} \quad 0,63$ & 0,66 & Q8 & 3,9 \\
$\begin{array}{l}\text { 5- O cuidado como } \\
\text { intervenção terapêutica }\end{array}$ & 0,63 & 0,66 & Q34 & 5,5 \\
$\begin{array}{l}\text { Todas as questões } \\
\text { Entre os 5 domínios }\end{array}$ & 0,71 & 0,72 & Q28 & 1,3 \\
\hline
\end{tabular}

Tabela 2 - Valores do Coeficiente Alpha de Cronbach para o instrumento EASC - Uberaba, MG, 2012

\begin{tabular}{|c|c|}
\hline DOMINIO & Coeficiente Alpha \\
\hline $\begin{array}{l}\text { 1- O cuidado como característica } \\
\text { pessoal humana }\end{array}$ & 0,62 \\
\hline $\begin{array}{l}\text { 2- } \mathrm{O} \text { cuidado como imperativo } \\
\text { moral }\end{array}$ & 0,66 \\
\hline 3- $O$ cuidado como afeto & 0,63 \\
\hline $\begin{array}{l}\text { 4- O cuidado como relação } \\
\text { interpessoal }\end{array}$ & 0,63 \\
\hline $\begin{array}{l}\text { 5- O cuidado como intervenção } \\
\text { terapêutica }\end{array}$ & 0,71 \\
\hline Todas as questões em conjunto & 0,74 \\
\hline Entre os 5 domínios & 0,45 \\
\hline
\end{tabular}

Tabela 3- Concordância entre testes e resultados da análise de reprodutibilidade - Uberaba, MG, 2012

\begin{tabular}{|l|llll|}
\hline DOMínIO & $\begin{array}{l}\text { Concordância } \\
\text { Observada } \\
(\%)\end{array}$ & $\begin{array}{l}\text { Coeficiente } \\
\text { de } \\
\text { Correlação } \\
\text { Intraclasse }\end{array}$ & $\begin{array}{l}\text { Confiabilidade } \\
\text { Brown }\end{array}$ & Intervalos \\
\hline $\begin{array}{l}\text { 1- O cuidado como } \\
\text { característica pessoal } \\
\text { humana }\end{array}$ & 95,6 & 0,72 & 0,84 & $0,76-0,90$ \\
$\begin{array}{l}\text { 2- O cuidado como } \\
\text { imperativo moral }\end{array}$ & 95,0 & 0,79 & 0,88 & $0,82-0,92$ \\
$\begin{array}{l}\text { 3- O cuidado como } \\
\text { afeto }\end{array}$ & 94,3 & 0,66 & 0,80 & $0,69-0,87$ \\
$\begin{array}{l}\text { 4- O cuidado como } \\
\text { relação interpessoal }\end{array}$ & 96,1 & 0,72 & 0,84 & $0,75-0,89$ \\
$\begin{array}{l}\text { 5- O cuidado como } \\
\text { intervenção } \\
\text { terapêutica }\end{array}$ & 95,1 & 0,63 & 0,78 & $0,66-0,85$ \\
\hline
\end{tabular}


Escala de Avaliação do Significado do Cuidar - EASC

Este questionário contém 44 afirmações. Depois de ler cuidadosamente cada uma, assinale a opção que melhor representa a sua opinião sobre o conteúdo da afirmação.

\begin{tabular}{|c|c|c|c|c|c|}
\hline Afirmativas & $\begin{array}{l}\text { Discordo } \\
\text { total } \\
\text { mente }\end{array}$ & Discordo & $\begin{array}{c}\text { Em } \\
\text { dúvida }\end{array}$ & Concordo & $\begin{array}{l}\text { Concordo } \\
\text { total } \\
\text { mente }\end{array}$ \\
\hline \multicolumn{6}{|l|}{ 1.Manter as próprias unhas cuidadas é importante } \\
\hline \multicolumn{6}{|l|}{$\begin{array}{l}2 . \text { O enfermeiro não deve expressar seus sentimentos } \\
\text { durante o cuidado }\end{array}$} \\
\hline \multicolumn{6}{|l|}{ 3.Estou sempre atenta às alterações da minha saúde. } \\
\hline \multicolumn{6}{|l|}{4.0 ato de cuidar exige competência } \\
\hline \multicolumn{6}{|l|}{ 5.Durmo satisfatoriamente, todos os dias } \\
\hline \multicolumn{6}{|l|}{$\begin{array}{l}\text { 6.Habitualmente, procuro fazer uma alimentação } \\
\text { equilibrada }\end{array}$} \\
\hline \multicolumn{6}{|l|}{ 7.Ao cuidar, o enfermeiro deve ser imparcial } \\
\hline \multicolumn{6}{|l|}{ 8.A meiguice precisa estar presente no ato de cuidar } \\
\hline \multicolumn{6}{|l|}{ 9.Cuidar é um ato de compaixão } \\
\hline \multicolumn{6}{|l|}{ 10.A aparência pessoal é importante para o cuidado. } \\
\hline \multicolumn{6}{|l|}{$\begin{array}{l}\text { 11.Quem que cuida deve saber o que o paciente pensa } \\
\text { sobre as próprias necessidades }\end{array}$} \\
\hline \multicolumn{6}{|l|}{$\begin{array}{l}\text { 12.A relação de empatia com o outro é importante no } \\
\text { cuidado }\end{array}$} \\
\hline \multicolumn{6}{|l|}{ 13.Cuidar do próprio corpo é importante. } \\
\hline \multicolumn{6}{|l|}{$\begin{array}{l}\text { 14. Cuidar envolve compromisso de um ser humano para } \\
\text { com outro }\end{array}$} \\
\hline \multicolumn{6}{|l|}{15.0 cuidado envolve afetividade } \\
\hline \multicolumn{6}{|l|}{ 16.Eu gosto de ser enfermeiro } \\
\hline \multicolumn{6}{|l|}{$\begin{array}{l}\text { 17.Cuidar envolve manejo adequado das situações que se } \\
\text { apresentam }\end{array}$} \\
\hline \multicolumn{6}{|l|}{$\begin{array}{l}\text { 18.O enfermeiro deve ser bem-humorado no desempenho } \\
\text { do cuidado }\end{array}$} \\
\hline \multicolumn{6}{|l|}{ 19.Cuidar envolve organização } \\
\hline \multicolumn{6}{|l|}{ 20.Cuidar envolve gentileza } \\
\hline \multicolumn{6}{|l|}{ 21.Cuidar envolve o ideal de servir } \\
\hline \multicolumn{6}{|l|}{ 22.Sendo impessoal o enfermeiro cuida melhor } \\
\hline \multicolumn{6}{|l|}{23.0 cuidado exige destreza } \\
\hline \multicolumn{6}{|l|}{24.0 cuidado envolve real interesse de quem cuida } \\
\hline 25.0 cuidado deve ser planejado & & & & & \\
\hline $\begin{array}{l}\text { 26.Para cuidar, o enfermeiro deve observar as normas } \\
\text { institucionais }\end{array}$ & & & & & \\
\hline $\begin{array}{l}\text { 27.A percepção que o cliente tem do enfermeiro interfere } \\
\text { no cuidado }\end{array}$ & & & & & \\
\hline 28.Educação deve fazer parte do cuidado de enfermagem & & & & & \\
\hline 29.Eu me cuido praticando atividade fisica & & & & & \\
\hline $\begin{array}{l}30.0 \text { bom cuidado depende da execução correta das } \\
\text { técnicas de enfermagem }\end{array}$ & & & & & \\
\hline $\begin{array}{l}31.0 \text { código de ética deve ser colocado em prática no } \\
\text { cuidar }\end{array}$ & & & & & \\
\hline 32.0 cuidado não tem preço & & & & & \\
\hline $\begin{array}{l}\text { 33.Para cuidar, o enfermeiro utiliza procedimentos } \\
\text { metódicos }\end{array}$ & & & & & \\
\hline 34.Habitualmente, utilizo o recurso da auto-medicação & & & & & \\
\hline $\begin{array}{l}\text { 36.Quem cuida precisa ter responsabilidade, respeito e } \\
\text { honestidade }\end{array}$ & & & & & \\
\hline 36.Ao cuidar o enfermeiro deve valorizar a comunicação & & & & & \\
\hline $\begin{array}{l}\text { 37.Considero os direitos e os deveres do cidadão ao } \\
\text { praticar o cuidado }\end{array}$ & & & & & \\
\hline 38.Cuidar envolve delicadeza & & & & & \\
\hline 39.A relação de confiança é necessária para o cuidado & & & & & \\
\hline $\begin{array}{l}\text { 40.Reservar algum tempo para o próprio lazer é } \\
\text { importante }\end{array}$ & & & & & \\
\hline 41. Quem que cuida deve considerar os valores do outro & & & & & \\
\hline 42.Quem cuida deve estar bem consigo mesmo & & & & & \\
\hline 43.O cuidado envolve perspicácia do enfermeiro & & & & & \\
\hline 44.Cuidar é sinal de solidariedade. & & & & & \\
\hline
\end{tabular}

